\section{A note on channel uncertainty in multidimensional signal detection tasks*}

\author{
RICHARD A. HEATH \\ McMaster University, Hamilton, Ont., Canada
}

Data reported by Lindsay et al (1968) were analyzed in terms of an extention of Kinchla's (1969) model of multiple observations in signal detection tasks, which takes into account the effects of channel uncertainty. The model was shown to fit this and other data with a high degree of accuracy. The implications of this analysis to theories of attention and temporal discrimination are discussed.

Lindsay, Taylor, \& Forbes (1968) conducted an experiment in which Ss were required to attend to one, two, or four dimensions of a multidimensional signal. Stimulus durations of 67,133 , and $200 \mathrm{msec}$ were employed, and $S$ reported which observation interval of a two-alternative forced-choice (2AFC) trial contained the larger value on one of the dimensions which was indicated to $\mathbf{S}$ by poststimulus cueing. Although $S$ might be monitoring more than one dimension per trial, only one dimension was chosen randomly for report. A signal detection analysis of the data showed that mean $d^{\prime}$ per dimension decreased with the number of dimensions requiring attention and increased with stimulus duration. In the two-dimensional monitoring conditions, performance did not depend on whether one or both sensory modalities were employed, thus providing evidence for a central attention mechanism.

Lindsay et al suggested that their results supported the idea that attention involves the distribution of the available information processing capacity among the dimensions monitored. Although Lindsay et al preferred a parallel model to account for their data, the tradeoff between stimulus duration and number of dimensions monitored might be accounted for by a $n$ attention-switching theory.

Franzen, Markowitz, \& Swets (1970) presented weak vibrotactile signals of $500 \mathrm{msec}$ duration to the index and middle finger using a $2 \mathrm{AFC}$ method. In the signal specified exactly (SSE) condition, Ss knew which finger or fingers would be stimulated during each block of trials. In the signal specified statistically (SSS) condition, Ss did not know which type of stimulus would occur on a given trial. In Condition SSE, there was no evidence of spatial summation between the two fingers, since

* This research was supported in part by Grant 214-7804 from the Division of Science and Engineering, McMaster University. performance with both fingers stimulated was no better than performance when the more sensitive finger was stimulated. Franzen et al suggested that their results supported a single-channel model of attention. They based their conclusion on the finding that performance in Condition SSS was superior when both fingers were stimulated and that performance when both fingers were stimulated was no better than performance on the more sensitive finger in Condition SSE.

Comparing the above two studies, it is obvious that the Ss' tasks are different in each case. Lindsay et al required $S s$ to attend to as many dimensions as possible on each trial. Since Franzen et al did not require $\mathrm{Ss}$ to identify the stimulus, the task was essentially disjunctive, so that a possible strategy would be to attend to the more sensitive finger on each trial. This explanation would account for performance in Condition SSE, and Franzen et al are not justified in assuming that Ss attended to only one finger in Condition SSS because they appeared to adopt this strategy in Condition SSE. Hence, the question of whether or not a single-channel mechanism imposes a limitation on performance is obscured by strategies

Kinchla (1969) proposed a model for temporal uncertainty in detection tasks that can be readily adapted to account for channel uncertainty effects. Assuming that signal detectability is invariant over observation intervals in a multiple-observation task, one obtains:

$$
d_{n, t}^{\prime}=d^{\prime} \operatorname{tn}^{-1 / 2}
$$

where $d_{n, t}^{\prime}$ is the detectability of a signal randomly located in $t$ of $n$ observation intervals and $d^{\prime}$ is the detectability of a signal presented in only one observation interval.

In a "multiple-look" task, the signal is present in all $n$ observation intervals, so that $t$ can be replaced by $n$ in Eq. 1:

$$
d_{n, n}^{\prime}=d^{\prime} n^{1 / 2}
$$
used by $\mathrm{S}$.
This result corresponds to the integration model for multiple observations of the same sinusoid, discussed by Green \& Swets (1966). Assuming that, within certain limits, increasing the signal duration is equivalent to increasing the number of looks at the signal, Eq. 2 summarizes the effect of signal duration on detectability.

In the temporal uncertainty task, a signal is present in only one observation interval, so that $t$ equals 1 and Eq. 1 becomes:

$$
d_{n, 1}^{\prime}=d^{\prime} n^{-1 / 2}
$$

Whereas, in a temporal uncertainty task, Ss might scan observation intervals serially, Ss performing a channel uncertainty task may adopt a similar strategy. If the channels are independent and contain equally detectable signals, Eq. 3 can be applied to account for the effect of channel uncertainty on signal detection performance. In this case, $\mathbf{n}$ refers to the number of channels being monitored.

Suppose that the signal on each channel has duration $t$ intervals of time, so that Eq. 2 may be rewritten as :

$$
d_{1, t}^{\prime}=d^{\prime} t^{1 / 2}
$$

where $d_{i, t}$ is the detectability of a signal of duration $t$ time units on a single channel. If $\mathbf{n}$ independent channels are being monitored, the detectability of a signal of duration $t$ time units on one of the $n$ channels is given by:

$$
d_{n, t}^{\prime}=d_{1, t^{-1 / 2}}^{\prime}=d^{\prime} t^{1 / 2} n^{-1 / 2}
$$

Squaring both sides of Eq. 5, we obtain:

$$
d_{n, t}^{\prime 2}=d^{\prime 2} \operatorname{tn}^{-1}
$$

and summing over $n$, the number of channels, gives :

$$
\sum_{n} d_{n, t}^{\prime 2}=d^{\prime 2} t=K
$$

where $K$ is constant for a constant signal duration. If we assume that $d_{n, t}^{\prime 2}$ is proportional to the information transmitted along each of $\mathbf{n}$ channels during $t$ time units (Taylor, Lindsay, \& Forbes, 1967), Eq. 7 implies that there is an upper limit to information processing capacity. If a logarithmic transformation of Eq. 6 is applied, we obtain:

$\log d_{n, t}^{\prime 2}=\log t-\log n+\log d^{\prime 2}$

so that a log-log plot of $d_{n, t}^{\prime 2}$ against $n$ for various values of $t$ should generate parallel lines with slope -1 . 


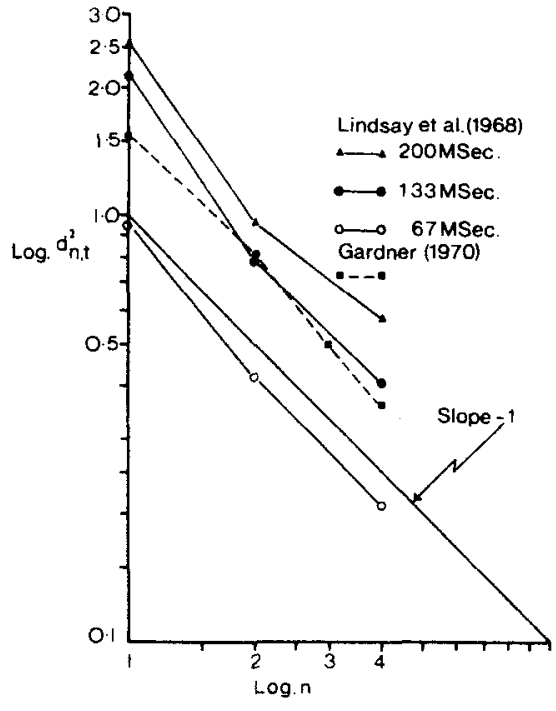

Fig. 1. A log-log plot of the Lindsay et al (1968) and Gardner (1970) data.

Lindsay et al (1968) provide data which can be used to test this prediction. Using the data pooled over Ss from Table 1 of their paper, a log-log transformation according to Eq. 8 was performed. The results of this analysis are shown in Fig. 1 . The $d^{\prime}$ values are mean values averaged across the various combinations of one, two, and four dimensions. Although the hypothesis of negative unit slope is not supported for all values of duration and number of dimensions monitored, the segments for multidimensional tasks at 67 and $133 \mathrm{msec}$ stimulus duration have slopes of -1.02 and -0.93 , respectively. If it is assumed that multidimensional tasks differ from unidimensional tasks, since the latter do not require an executive process to control time-sharing of inputs which might reduce the available information processing capacity, then it is reasonable to limit the applicability of the model in its simplest form to multidimensional discrimination tasks. Also, it is quite likely that with a 200-msec signal duration, all the information contained in the signal may not be required to reach a decision, since, as Fig. 2 shows, the model tends to overestimate performance at longer stimulus durations. The solid lines in Fig. 2 were obtained by choosing a value of $d^{\prime}$, the only parameter of the model estimated from the data, which minimized the chi-square value between the observed and predicted values of $d_{n, t}^{\prime}$ from $E q .5$. Hence, the model provides a satisfactory fit to these data for signal durations not exceeding $133 \mathrm{msec}$. The model also appears to be quite robust, since the assumptions of equally detectable signals and independent stimulus dimensions are probably not satisfied by the Lindsay et al data.

Lindsay et al (1968) pointed out that their data cannot be accounted for by a single-channel mechanism which only attends to one stimulus dimension per trial. Assuming that Ss are unbiased in their distribution of attention, the probability that attention is directed towards the dimension cued for report is $n^{-1}$, where $n$ is the number of dimensions to be monitored. Hence, the detectability of a signal dimension for an $n$-dimensional stimulus of duration $t$ time units is given by:

$$
d_{n, t}^{\prime}=d^{\prime} n^{-1} t^{1 / 2}
$$

Squaring and applying the log-log transformation gives:

$\log d_{n, t}^{\prime 2}=\log t-2 \log n+\log d^{\prime 2}(10)$

so that a log-log plot of $d_{n, t}^{\prime 2}$ against $n$ for various values of $t$ should generate parallel lines with slope -2 . This consequence of the assumption of a single-channel attention mechanism is not substantiated by the data, as Fig. 1 clearly demonstrates. Using a similar argument and assuming that $\mathrm{Ss}$ can attend to no more than two dimensions per trial, Eq. 10 should fit the data to two- and four-dimensional tasks. However, a slope of -2 is clearly not obtained, so that even at the shortest stimulus duration, $67 \mathrm{msec}$, Ss were attending to at least three dimensions of the signal. Hence a switch of attention must occur at least once every $22 \mathrm{msec}$, a value well below some experimental estimates of switching times (Kristofferson, 1967). Even if Ss could attend to intramodality dimensions simultaneously, it is difficult to reconcile this with the finding that performance was not affected by the combination of dimensions, but only by their number.

One objection to the above argument is that Ss were able to retrieve information from some representation of the signal in memory, so that the effective duration of the signal is longer than its physical duration. However, if this is so, it is difficult to explain why performance on two-dimensional tasks for a duration of $67 \mathrm{msec}$ is the same as performance on four-dimensional tasks for a duration of $133 \mathrm{msec}^{1}$ Moreover, no significant effect of delay of cue was obtained (Lindsay, 1970).

Data obtained by Gardner (1970) can also be analyzed so that the goodness of fit of the model can be evaluated. Stimuli consisted of consonants typed on an imaginary

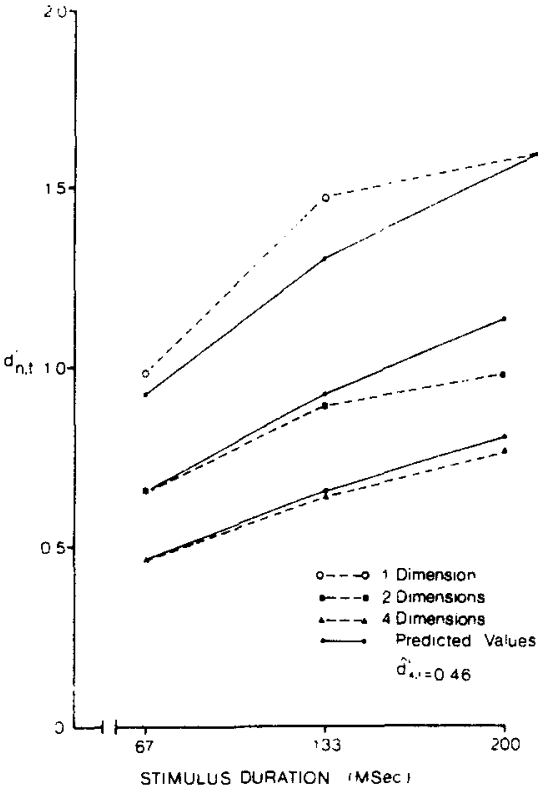

Fig. 2. Observed and predicted values of $d_{n, t}^{\prime}$ as a function of stimulus duration.

square, $1.8 \mathrm{deg}$ of visual angle apart. A 32-card deck was prepared for each of four array sizes-one, two, three, or four consonants. Within each deck, " $N$ " and " $P$ " were used as target letters equally often and appeared in each of the four possible spatial locations with equal frequency. On each trial, S reported which of the two possible target letters was present, the task being a $2 \mathrm{AFC}$ similar to that used by Estes \& Taylor (1964).

Percentage of correct responses for each array size were transformed into $\mathrm{d}^{\prime}$ measures and substituted into Eq. 8, with $t$ being set arbitrarily at 1 . The prediction of a linear relationship with slope -1 is well substantiated by the data plotted in Fig. 1. An interpretation of these data in terms of channel uncertainty is made difficult because, within each array size (equated here with the number of visual spatial channels), the positions of the letters were random. Hence, the effect of channel uncertainty does not operate at the periphery of sensory systems, but at some point where information from several sources converges on to a limited capacity processor. Hence, for the Gardner data, the concept of attention switching is irrelevant.

Recently Earle \& Lowe (1971) found further support for Kinchla's model. Employing a 2AFC task with signals of $85 \mathrm{msec}$ duration, Earle and Lowe investigated the effect of channel uncertainty on the detectability of auditory and visual signals. In the first condition, Ss detected auditory signals alone; in the second condition visual signals alone; 
in the third condition, however, Ss were required to detect randomly intermixed auditory and visual signals. Earle and Lowe found a significant decrement in detectability for both auditory and visual signals as a result of channel uncertainty. The decrement was close to that predicted by Kinchla's model of channel uncertainty.

The above review has shown that the model for channel uncertainty can account for the results of diverse studies in discrimination and recognition. Although some of the assumptions of Kinchla's original formulation may not be met by the data, the close fit suggests that exact satisfaction of these demands may not be necessary. In particular, this type of model highlights the futility of adhering to either one of the parallel and serial views of human information processing (Townsend, 1971), since with a sufficiently short switching time both views can be satisfied by this model. The tradeoff between stimulus duration and channel uncertainty predicted by the model is strictly additive and suggests that time may be a dimension of a stimulus independent from other sensory dimensions. This finding should have important implications for theories of time discrimination, since it is necessary to measure the temporal dimension of a stimulus independent of other stimulus characteristics in order to fit a purely temporal model to data. The present model suggests that this formidable task may be indeed possible.

\section{REFERENCES}

EARLE, D. C. \& LOWE, G. Channel, temporal, and composite uncertainty in the detection and recognition of auditory and visual signals. Perception \& Psychoph ysics, 1971, 9, 177-181.

ESTES, W. K. \& TAYYLOR, H. A. A detection method and probabilistic model for assessing information processing from brief visual displays. Proceedings of the National Academy of Sciences, 1964, 52, 446-454.

FRANZEN, O., MARKOWITZ, J., \& SWETS, J. A. Spatially-limited attention to vibrotactile stimulation. Perception \& Psychophysics, 1970, 7, 193-196.

GARDNER, G. T. Spatial processing characteristics in the perception of brief visual arrays. Human Performance Center. Department of Psychology, University of Michigan, Ann Arbor, Technical Report No. 23, August 1970.

GREEN, D. M., \& SWETS, J. A. Signal detection theory and psychophysics. New Y ork: Wiley, 1966.

KINCHLA, R. A. Temporal and channel uncertainty in detection: A multiple observation analysis. Perception \& Psychophysics, 1969, 5, 129-136.

KRISTOFFERSON, A. B. Attention and psychophysical time. Acta Psychologica, $1967,27,93-100$.
LINDSAY, P. H. Multichannel processing in perception. In D. I. Mostafsky (Ed.), Attention: Contemporary theory and $a n a l y s i s$. N e w Y O $\mathrm{kk}$ : Appleton-Century-Crofts, 1970.

LINDSAY, P. H., TAYLOR, M. M.. \& FORBES, S. M. Attention and multidimensional discrimination. Perception \& Psychophysics, 1968, 4, 113-118.

TAYLOR, M. M., LINDSAY, P. H., \& FORBES, S. M. Quantification of shared capacity processing in auditory and visual discrimination. Acta Psychologica, 1967, 27, 223-229.

TOWNSEND, J. T. A note on the identifiability of parallel and serial processes. Perception \& Psychophysics. $1971,10,161-163$.

\section{NOTE}

1. Suppose that at the offset of the signal, a memory trace decays at a rate independent of stimulus duration. If $I$ is the stimulus intensity, $D$ the stimulus duration, and $\lambda$ the rate of decay of the trace, then the total information content of the stimulus can be represented as: $H=$ $I(D+1 / \lambda)$. Hence, for an n-dimensional signal of duration $t$ time units, the average information per dimension is given by: $\bar{H}_{n, t}$ $=(I / n)(t+1 / \lambda)$. Let the average information in a 67-msec signal on two dimensions be $\vec{H}_{2,1}$ and the average information in a 133-msec signal on four dimensions be $\overline{\mathrm{H}}_{4,2}$. Now, $\overline{\mathrm{H}}_{2,1}-\overline{\mathrm{H}}_{4,2}=0.5 \mathrm{I}(\mathrm{t}+1 / \lambda)-$ $0.25 \mathrm{I}(2 \mathrm{t}+1 / \lambda)=0.25 \mathrm{It} / \lambda$. But, if the result can be explained in terms of such a memory mechanism, $\overline{\mathrm{H}}_{2,1}-\overline{\mathrm{H}}_{42}=0$. Hence, such an explanation seems unlikely. 